\title{
477 COM902 (ANTI-TIGIT ANTIBODY) MONOTHERAPY - PRELIMINARY EVALUATION OF SAFETY, TOLERABILITY, PHARMACOKINETICS AND RECEPTOR OCCUPANCY IN PATIENTS WITH ADVANCED SOLID TUMORS (NCT04354246)
}

${ }^{1}$ Ecaterina Dumbrava*, ${ }^{2}$ Drew Rasco, ${ }^{2}$ Amita Patnaik, ${ }^{3}$ Daniel Vaena, ${ }^{2}$ Kyriakos Papadopoulos, ${ }^{3}$ Adam ElNaggar, ${ }^{4}$ Adeboye Adewoye, ${ }^{4}$ Robina Smith, ${ }^{4}$ Pierre Ferré, ${ }^{4}$ llan Vaknin, ${ }^{5}$ Srinivas Devarakonda, ${ }^{6}$ Manish Sharma. ${ }^{1}$ MDACC, Houston, $T X$, USA; ${ }^{2}$ START - San Antonio., San Antonio, TX, USA; ${ }^{3}$ West Cancer Center and Rsch Institute., Memphis, TN, USA; ${ }^{4}$ Compugen USA Inc., San Francisco, USA; ${ }^{5}$ Ohio State University, Columbus, OH, USA; ${ }^{6}$ START - Midwest., Grand Rapids, MI, USA

Background COM902 is an IgG4 fully human high-affinity monoclonal antibody, inhibitor of TIGIT (T cell Ig and immunoreceptor tyrosine-based inhibitory motif [ITIM] domain) binding to poliovirus receptor (PVR). TIGIT blockade by COM902 was shown to enhance anti-tumor immunity in preclinical models. We hypothesized that COM902 as monotherapy will have an acceptable safety and tolerability profile in subjects with advanced solid tumors.

Methods Utilizing an accelerated titration and $3+3$ study design we enrolled 18 patients (pts) at the following COM902 doses $0.01,0.03,0.1,0.3,1,3$ and $10 \mathrm{mg} / \mathrm{kg}$ IV Q3 wks. Key primary objectives were to evaluate the safety, tolerability (CTCAE v5.0), to characterize the pharmacokinetics (PK) and to select a recommended dose for expansion (RDFE). An exploratory objective was evaluation of peripheral receptor occupancy (RO). Key inclusion criteria: Age $\geq 18$ yrs, histologically/cytologically confirmed advanced malignancy who have exhausted all available standard therapy or not a candidate for standard therapy. Patients with performance status ECOG $0-1$, prior ICI permissible. Dose-limiting toxicities (DLTs) were evaluated within a 21-day window in the 1st cycle of dose escalation.

Results In the safety population $[\mathrm{N}=18], 12$ pts reported treatment emergent adverse events (TEAEs). The most frequent TEAES $[\geq 2$ pts] were fatigue 7 pts (39\%) all G1/2, diarrhea 3 pts (17\%) all G1/2. Two pts reported DLTs deemed related to study drug, a pt with G2 nausea (single pt cohort, $0.01 \mathrm{mg} / \mathrm{kg}$ ) and a pt with $\mathrm{G} 3$ atrial fibrillation $(1 \mathrm{mg} / \mathrm{kg})$. Serious adverse events were reported in 2 pts, 1 pt with atrial fibrillation (deemed by the investigator as possibly related to COM902) and 1 pt with spinal cord compression (deemed by the investigator as unrelated to COM902, related to disease). Preliminary PK profiles were generally dose proportional and peripheral RO above $90 \%$ was reported from $0.1 \mathrm{mg} / \mathrm{kg}$ dose. Conclusions COM902 has an acceptable safety, tolerability and PK profiles. A COM902 $3 \mathrm{mg} / \mathrm{kg}$ IV Q3 wks is the RDFE. Enrollment into combination cohort (COM902 + COM701), for evaluation of safety/tolerability at the RDFE of both study drugs, combination dose expansion (COM902 + COM701) in pts with HNSCC, NSCLC and CRC-MSS and COM902 monotherapy dose expansion (pts with multiple myeloma) all at the RDFE of study drug(s) are planned. Data cut June 28, 2021.

Trial Registration NCT04354246

Ethics Approval The study obtained approval from IRBs of the participating clinical trial sites. The study participants gave informed consent before taking part.o 0002: MOD01006350 (START)o 0003: 20202320 (West Cancer Center)o 0012: 2020-0195 (MDACC)o 0013: MOD01006350 (START midwest)o 0014: 20202320 (OSU) 